\title{
CONTINUIDAD Y DISCONTINUIDAD EN LA CONSTITUCION DE 1876
}

\author{
POR \\ JOSE MANUEL PEREZ-PRENDES Y MUÑOZ DE ARRACO \\ Catedrático de Historia de las Instituciones Políticas y Administrativas \\ de España en la Universidad Complutense
}

Deseo sintetizar en este trabajo alguna reflexión sobre la Constitución de 1876 , preferentemente orientada a sugerir ciertas valoraciones sobre su significación dentro del totum de nuestro sistema jurídico constitucional, más que referirme sólo a la hermenéutica de su propio articulado. A lo largo de la redacción de estas notas he ido percibiendo cierta presencia de un rasgo no buscado de antemano: la contradicción con determinados enfoques, habitual no unánimemente, asimilados por la doctrina y la historia políticas de nuestro constitucionalismo. Debo disculparme por ello y subrayar desde ahora mi siempre procurada actitud de revisión futura.

\section{A) EL PROCESO HISTORICO}

La Constitución promulgada en 30 de junio de $1876^{1}$ se sitúa en el segundo período de los que entiendo sigue el proceso de la historia del régimen constitucional español, período al que en otro lugar he llamado «de revisiones profundas» ${ }^{2}$. En efecto, frente a la idea, que estimo simplista, de resaltar los epifenómenos históricos para concluir caracterizando aquel proceso como una sucesión de cambios bruscos y violentos, en los que el Derecho no es un factor de consolidación ni sosiego, me parece más ajustado a una línea de realidad profunda de las cosas comenzar afirmando la tesis de que incluso a lo largo del siglo XIX se darán condiciones en las que aquella forma de sociedad a la que desde fines del xviII llamamos «Antiguo Régimen», podrá pervivir como última y casi impercibida (pero no irreal) ratio de la organización políticosocial española. Los cambios constitucionales hasta 1869 resultaron, quizá contra la voluntad de sus impulsores, los precisos para que la perduración citada fuese posible en alguna medida en cada momento. Bajo la idea de su

1 Diego Sevilla Andrés, Constituciones y otras leyes y proyectos políticos de España, 2 vols., Madrid, 1969. El texto de la Constitución de 1876, en el I, pág. 597.

${ }_{2}$ José Manuel Pérez-Prendes, Curso de Historia del Derecho español, 2." ed., Madrid, 1978, págs. 769 y sigs. 
continuidad, esas variantes estratégico-jurídicas (dicho sea con humor) las podemos ordenar nosotros hoy en tres etapas que unifican, articulándose, el esqueleto de nuestro sistema jurídico constitucional.

Un primer período (1812-1834) de radicales (en su sentido etimológico) contradicciones, entendidas como lo haría un filósofo que diferenciase entre «contrarios» y «contradictorios». Quiero decir que principios jurídico-políticos básicos, perfectamente incompatibles entre sí, van a intentar desplazarse mutuamente durante esos años. La «agonía del Antiguo Régimen», entendiendo por ella, como quería Unamuno antes que Heidegger, las ansias entre la vida y la muerte, durará entre nosotros en su fase más intensa, que coincide con este primer período propuesto, veintidós años, plazo que contrasta con los cuarenta meses (1789-1793) que ostenta el fenómeno en la historia francesa. Cierto es que también en el vecino país existieron perduraciones que alargaron en formas residuales, hasta 1850 más o menos, las huellas de un sistema político cada vez más vencido en rupturas decisivas. Pero toda pervivencia meramente residual carece de vitalidad latente para ser basamento de revisiones fundamentales. A veces, y a lo sumo, imponen la lentitud en la consumación revolucionaria, pero no poseen fuerza para imprimir su sello al tiempo en que viven ${ }^{3}$. En cambio entre nosotros esa larguísima agonía supuso primero, y pese a las evidentes transformaciones en temas concretos, como el régimen señorial, el fracaso del esfuerzo por «abolir la forma antigua de la sociedad» ${ }^{4}$.

Desde ahí se abre una perspectiva nueva que nos permite contemplar en un segundo período, desde 1834 a 1868, cómo se produce una posibilidad de perduración real para esa «forma de sociedad» que es el «Antiguo Régimen», aun podado de muchas de sus instituciones típicas. Perduración que ya no se afirma como hasta entonces por la vía contradictoria ni por ningún otro camino de negaciones frontales y directas, sino que va a resultar latente, pese a que podría designarse este nuevo tiempo como etapa de «consolidación constitucional». Admitiría esa designación recordando la frase de Posada, según la cual, «a partir de la Constitución de 1837, las acciones y reacciones políticas tendrán como supuesto una Constitución» ${ }^{5}$. No se me oculta que precisamente 1837 es, por ejemplo, para Artola, el momento en que concluye el proceso iniciado en 1808 para conseguir «la pretensión de restaurar el Antiguo Régimen, que para (1837) habrá dado paso al régimen liberal» ${ }^{6}$. Pero si se leen con atención sus palabras, se observará que se refiere básicamente a «la incapacidad de conseguir una decisión militar» restauradora, incapacidad puesta de manifiesto de modo bien gráfico con el fracaso de la expedición real carlista de ese año. Así, pues, Artola está aludiendo al fin de las reacciones directas que, para mí, tipifican el período anterior, al que he llamado de «contradicciones radicales» y del que no podría poner inconveniente alguno para que se

${ }^{3}$ Pierre Goubert, L'ancien regime, Paxís (Colin), 1969, cap. I. Existe una traducción española, absolutamente detestable.

${ }_{4}^{4}$ Alexis de Tocqueville, El Antiguo Régimen y la revolución, Madrid, 1969, pág. 33.

5 Adolfo Posada, Tratado de Derecbo politico, vol. II, Derecho constitucional comparado de los principales Estados de Europa y América, Madrid, 1935, págs. 258 y sigs.

${ }^{6}$ Miguel Artola, Antiguo Régimen y revolución liberal, Madrid, 1978, pág. 159. 
considere zanjado en 1837. Lo que ya me cuesta más trabajo es no matizar mucho los términos en que desde esa fecha tiende a consolidarse entre nosotros, según la interpretación de los historiadores actuales, una de las llamadas «revoluciones liberales burguesas» ${ }^{7}$.

Atendiendo a un planteamiento específico de historia constitucional, creo imprescindible poner el acento en otro rasgo: en el hecho de que el eje doctrinal vigente en este período es el doctrinarismo y que, mientras esa concepción subsiste, lo que realmente vive con ella es un vacío, vacío que en esta segunda fase del proceso constitucional (1834-1868) es propicio a prolongar esencias políticas del antiguo régimen. La «consolidación constitucional» es así más consolidación formal que real, aun cuando tenga una innegable trascendencia, si se considera el signo atormentado del período anterior. Dicho esto, debería subrayarse además que ahora se buscará superar el conflicto planteado en esa etapa precedente mediante la simple apelación a técnicas de formalismo jurídico. Esto es, divorciando en parte el Derecho de la realidad, aplicando coberturas normativas (que irán poco más allá de su «formulación») a la referida forma de sociedad a la que llamamos Antiguo Régimen, que, en fin de cuentas, encuentra condiciones para persistir, dada la carencia de eficacia transformadora de la mera norma. La conciencia que alcanzaron los sucesivos protagonistas de cuál era la última y profunda línea de deslizante efecto de sus reformas es cuestión que no puedo abordar aquí, pero quizá haya de apuntarse que fuese nula o muy escasa, ya que para la formación de esa conciencia hace falta perspectiva, y ella no se suple con el acumulo de documentación que su posición política les proporcionaba. Pero como veremos, habrá con Giner de los Ríos algún lúcido denunciante de la trampa doctrinarista ${ }^{8}$.

El papel que en este segundo período de nuestro proceso constitucional juega, en el sentido apuntado, el formalismo jurídico queda en nítido resalte cuando se comparan textos como las Constituciones de 1837 y 1845 . Siendo la primera, en fin de cuentas, una síntesis impregnada en su articulado del espíritu de la de 1812, bastan unos cambios en las frases iniciales para que su genuino contenido doceañista se adapte a la única idea imprescindible para la continuidad real del Antiguo Régimen: la afirmación de que el rey y las Cortes son «partícipes en el ejercicio de la soberanía» ${ }^{9}$, es decir, el mencionado constitucionalismo doctrinario. Con esa simple técnica se desdentará el primer escollo que en el nuevo tiempo se ofrece a la continuidad del Antiguo Régimen: la Constitución de 1837. Ahora bien: tampoco puede dejar de anotarse que, pese a todo, los pasos meramente formales, típicos de este instante, son pasos necesarios que posibilitan un futuro más hondamente transformador.

El sexenio revolucionario (1868-1874), del que habría que considerar como preludio el espacio esparterista de 1854-1856, es, creo, interpretable como el

\footnotetext{
7 Sobre la cuestión de la revolución burguesa en España, cfr. ahora Josep Fontana, Sobre revoluciones burguesas y autos de fe, en «Mientras tanto», 1 (1979), con revisión crítica de la bibliografía reciente. El concepto general en Marx y Engels, Manifiesto del partido comunista, en Obras escogidas, I, Madrid, 1975, pág. 27.

${ }^{B}$ Francisco Giner de los Ríos, La política antigua y la política nueva, en Estudios jurídicos y políticos, vol. $\mathrm{V}$ de las Obras completas, Madrid, 1921, págs. 63-189.

${ }^{9}$ Posada, op. cit., supra, nota 5, pág. 288.
} 
episodio que real, y no sólo formalmente, se propone implantar una forma de sociedad diferente a la que conocemos como «Antiguo Régimen». Por eso, a partir de ahí, entiendo que se dibuja el tercer período en nuestro discurso histórico-constitucional, al que podría designarse con la rúbrica general de ser el de las «revisiones profundas» (1868-...). La dicotomía entre «reforma» o «revolución» (a veces más púdicamente «ruptura»), entendidas como realidades totales históricamente alcanzables en cuanto alternativas en un momento concreto, es una trampa política, ya que no existe nunca una «ruptura» plena con el pasado, como enseña tozudamente la historia ${ }^{10}$. Toda revolución pasa por un cierto número de rupturas, sucesivas y parciales, que implican, por tanto, continuidades no menos parciales y sucesivas. La conciencia de esta realidad supone que al situar en 1868-1874 la crisis decisiva del Antiguo Régimen no me limito a proponer con más o menos ingenio un simple cambio de fecha, rebajando algo menos de sesenta años (de 1812 a 1868) el momento generalmente admitido para situar cronológicamente la pérdida de vigor, significativa, no transitoria, del Antiguo Régimen en España. La verdad es que, a mi entender, en 1868 el cosmos de ese Antiguo Régimen estaba mucho más presente que ausente en nuestra patria. Pierre Goubert ha sabido resumir la complejidad de sus sistemas en unas líneas breves justamente famosas por su riquísimo contenido y que importa mucho leer despacio:

«Económicamente se caracteriza por la lentitud de sus relaciones, el predominio de la agricultura, la pequeñez de la metalurgia en una industria de por sí secundaria, la casi nulidad del sistema bancario. Demográficamente... por los altos niveles, en conjunto de nupcialidad, fecundidad y mortalidad, y la perduración de epidemias y hambres. Políticamente... por la diversidad jurídica, lingüística y administrativa... Mentalmente, marcado por la confusión de la creencia en supersticiones y fervor cristiano, frecuente analfabetismo, vida provincial y local extremadamente aislada, concepción habitualmente débil y a veces nula del Estado, de la Nación, de la Patria, salvo en la adoración del monarca o la presencia física del peligro» ${ }^{11}$.

Basta recordar el horizonte social de los galdosianos Episodios nacionales, o de las Memorias de un hombre de acción, de Pío Baroja, o de La Corte de los milagros, de Valle-Inclán, o, en fin, de algunas novelas de Blasco Ibáñez; baste invocar la Guerra de la Independencia o el auténtico cariño popular al siniestro Fernando VII para admitir cuánto de ese ambiente espeso y contradictorio pervivía en España y en épocas constitucionales aun tardías. Pese a las declaraciones formales de una Constitución tras otra, muy pocas de las que pueden considerarse rupturas decisivas con ese mundo se habían producido entre nosotros hasta $1868^{12}$, e incluso aquellas áreas en que se registran logros parciales para una nueva forma de sociedad, como la industrialización o los ferrocarriles

10 Sobre el tema de la continuidad y discontinuidad en la Historia jurídica en general, cfr. José Manuel Pérez-Prendes, op. cit, supra, nota 2, págs. 130 y sigs.

${ }_{11}$ Op. cit., supra, nota 3 , pág. 31 del vol. I, en la trad. española. Los subrayados y la versión ofrecida aquí son míos.

${ }_{12}$ Goubert, op. cit., págs. 29-30. 
que aceleran los transportes, se nos presentan como esfuerzos que se agotan por la incapacidad del sistema político-social en llegar a concebir frente a horizontes rutinarios un régimen de economía nacional que dividiese el trabajo y acoplase resultados ${ }^{13}$. Tampoco el establecimiento de una estructura bancaria, que en todas partes se gesta lentamente durante el siglo XIX, se consolidará en España hasta bien entrado el $\mathrm{xx}$, y por lo que respecta a la Banca privada, sufrirá colapsos retardatarios hasta $1874^{14}$. En otros aspectos la indiferenciación con el Antiguo Régimen era aún más intensa. No se logró la aceptación social del sistema de servicio militar, siendo el tema de las quintas y las compañías de seguros contra ellas uno de los puntos más sensibles a la opinión pública; así lo muestra la demagógica apelación a tal cuestión a la hora de recabar apoyo popular a los candidatos al poder, como en el caso de Prim ${ }^{15}$. Las unificaciones lingüística y jurídica del país acometidas en Francia y España culminaron en el país vecino, pero no entre nosotros, donde la centralización apenas fue más allá de simplificar y uniformizar la Administración. Cierto que esas unificaciones se iniciaron mucho antes de la Revolución, pero recuérdese, de coincidencia con Tocqueville, que en Francia los revolucionarios asumieron ese legado en la medida en que les permitía instaurar «un orden social y político más uniforme» ${ }^{16} \mathrm{y}$ que lo potenciaron con instrumentos de rotunda eficacia para la unidad nacional, como el Código civil de Napoleón, mientras que en España una pieza similar sólo aparece en 1889, y mutilado su ámbito de vigencia por la presencia de los derechos forales.

Dentro de la elemental prudencia metodológica que aconseja no formular proposiciones demasiado inelásticas, cabría, en mi opinión, entender la Gloriosa Revolución Nacional de 1868 como el punto en el cual la conciencia de que el sistema político-social propio del Antiguo Régimen seguía presente y operante alcanza el punto más alto durante todo el siglo xIx, y por ello emprenden los revolucionarios la tarea no ya de cambiar sólo y una vez más la Constitución, cuanto de construir un orden jurídico nuevo que circule por las venas del sistema social ${ }^{17}$. A partir de aquí se iniciarán al menos dos revisiones profundas de trascendental importancia.

13 Jordi Nadal, El fracaso de la revolución industrial en España (1814-1913), Madrid, 1975, pág. 227; Gabriel Tortellá, Los orígenes del capitalismo en España, Madrid, 1973, páginas 319 y sigs.

${ }^{14}$ La Banca española en la Restauración, I. Política y finanzas, Madrid, 1974, especial. mente los estudios de Rafael Anes y Pedro Tedde sobre el Banco de España y la Banca privada, respectivamente.

${ }_{15}$ El joven investigador Agustín Albaladejo, desaparecido prematuramente, no llegó a publicar un importante estudio sobre el general Prim, en el que trabajó incansablemente varios años reuniendo una importante masa documental para su redacción, que tenía iniciada en el momento de su inesperada muerte. Varias veces habíamos comentado el tema de las quintas, tema sobre el que llegó a publicar algún artículo en revistas militares, en tono divulgatorio, usando del material reunido. Véase además todavía Nuria Sales, Sobre esclavos reclutas y mercaderes de quintos, Barcelona, 1974.

${ }_{16}$ Tocqueville, op. cit., supra, nota 4 , pág. 48.

${ }_{17} \mathrm{La}$ conocida monografía de Carro, que tuvo en su fecha cierto interés, no satisface la actual necesidad de conocer en sus fundamentos el orden jurídico proyectado por la Gloriosa. Una investigación en esa dirección ha sido acometida por el profesor de la Universidad de Granada don José Garrido Arredondo. 
La primera de ellas afectará a la Monarquía, que, como forma de gobierno, no había sido puesta en cuestión ni en el período de contradicciones radicales ni en el de consolidación constitucional a los que antes me he referido. Parece que tampoco lo es directamente ahora, con el primer alborozo del sexenio. Pero la realidad es que se logrará desviar la contestación antimonárquica con un hábil juego de planteamientos dinásticos. Se manifestaba así el lento pero continuo deterioro de la institución, que en última instancia el doctrinarismo había desgastado, para intentar mantener los sistemas de poder socio-político del Antiguo Régimen, contra los que luchó la Revolución y que, en fin de cuentas y por ironía de la Historia, eran en vida de aquél los más netos enemigos de cualquier corona poderosa. La actitud promonárquica de Prim jugará la baza de mantener la institución cambiando la dinastía, con el resultado conocido, y aún cabe que hoy nos sigamos preguntando si su distinción entre los dos conceptos fue captada como él la entendía por otros también monárquicos y qué consecuencias tuvo, si no fue así, esa mezcla acrítica de contenidos mentales en algunos sectores de opinión. Sea como fuere, su fracaso, quizá inevitable, quizá forzado (no cabe aquí la historia contrafactual), abrió las consecuencias de la identificación contenida durante decenios, pero ya planteada sin más dilación posible entre Monarquía y Antiguo Régimen, identificación que lleva al primer republicanismo como vía para lograr el destierro de aquél.

La segunda revisión pasa por otra médula del problema de los tránsitos al constitucionalismo. Ya he dicho cómo la unificación lingüística, jurídicoprivada y administrativa que Francia consigue en un lentísimo pero irrevertido proceso no se consolidó de igual modo en España. La Primera República se enfrentó con el hecho. Para acometer esta dificultad caben tres soluciones agrupables en dos mundos jurídico-políticos diversos. Al espíritu del Antiguo Régimen convienen tanto la opción de reemprender los esfuerzos centralizadores (incluyendo el reducir las libertades que sea necesario cercenar y establecer la mecánica represiva, no pequeña, que se estime apropiada) como la fórmula de autonomías desiguales, de contenido variable, casi como en la concepción stamleriana del Derecho natural. Por enfático que se quiera hacer el rechazo de este rasgo que establece un cordón umbilical entre Estados autonómicos (como el español, según la Constitución de 1978) y el Antiguo Régimen, es evidente que la «diferenciación» entre comunidades nace en gran medida de la vida provincial y local aislada, que con justicia Goubert identifica como uno de los ejes del viejo sistema. Ese aislamiento ha engendrado muchas veces la conciencia firme de diferencias (piénsese en Sabino Arana o Blas Infante), que el historiador no aislado sabe que no existen como tales, pero que, una vez emocionalizadas y escolarizadas, son muy esquivas a la crítica. Añádase que precisamente por y sobre esos presupuestos la metamorfosis disgregatoria es uno de los elementos que siempre asoma en el mundo de los países europeos anterior a la Revolución. Dados los planteamientos ideológicos no ya de la República, sino de todo el sexenio, la única solución que así se podía proponer al duro crujido de la realidad española que los hechos presentaron, no podía ser otra que un federalismo puesto en práctica con rapidez y seguridad por un gobierno poderoso, no por serlo contra otras fuerzas políticas, sino por resultar 
avalado por todas ellas. Pero si en 1874 se halló la fórmula, desfalleció la firmeza. Dejando ahora a un lado la todavía actual sensación de cuasi terror ante la posibilidad de un federalismo español, no se puede negar que la desaparición final del Antiguo Régimen pasaba en buena medida por los términos en que el sexenio intentaba aplicar unas veces la moderación y otras la coherencia.

\section{B) LAS CONTINUIDADES Y LAS DISCONTINUIDADES}

La Constitución de 1876 se nos presenta inicialmente comprometida por el proceso histórico a cuya significación acabo de apelar. $Y$ por eso ofrece un claroscuro de relaciones, tanto con los esfuerzos por apartar definitivamente el Antiguo Régimen, como con las actitudes que permitían conservar las huellas de ese régimen en cuanto forma de sociedad. Vamos ahora a considerar de modo breve alguna (fundamentalmente dos) de esas continuidades de muy heterogéneos signos, pues de un lado se aprecia la huella de la tradición doctrinaria, que en el período de «consolidación constitucional» sirve en todo caso a la perduración de formas propias del Antiguo Régimen, y desde otra óptica no puede descartarse una clara presencia de rasgos heredados del sexenio. No hubo apenas contrapeso entre ambas continuidades y el barco se escoró del lado de los más poderosos influjos, es decir, los primeros.

Es de sobra conocido el carácter doctrinario de la Constitución de 1876, basada en el pacto entre los que se consideran titulares de la soberanía, el rey y las Cortes:

«Don Alfonso XII... en unión y de acuerdo con las Cortes actualmente reunidas, hemos venido en decretar y sancionar la siguiente Constitución.»

Ya he indicado reiteradamente cómo el doctrinarismo del período de consolidación constitucional sirvió en definitiva para prolongar las huellas de los sistemas de poder incardinables en el Antiguo Régimen, pese a que se suele indicar el año de 1837 como fecha símbolo de su liquidación. En esa época cambiaron las tácticas restauradoras, que de violentas se hicieron encubiertas, con algunas oscilaciones y concesiones resueltas en la práctica con los formalismos a los que antes apunté.

En la primera parte de un trabajo fechado casi a todo lo largo del sexenio (de 1868 a 1872), don Francisco Giner de los Ríos formuló una lúcida crítica del doctrinarismo, aun no dejando de reconocer los logros concretos que debían anotarse en su haber ${ }^{18}$. La idea central que Giner desenvuelve es denunciar la carencia de una atención por parte del doctrinarismo «al fin del Estado», lo que implicará su ignorancia de «una idea precisa y determinada del Estado», idea que es la «fundamental de la política». Ese vacío impide dotar al Derecho que el Estado engendra, de una «base esencial, ética, interna, y confundiéndolo con la voluntad, que sólo es su instrumento y medio de realiza-

${ }^{18}$ Op. cit., supra, nota 8 , pág. 89 . 
ción», reduce al «Estado a un mecanismo más o menos artificioso». Una y otra vez a lo largo de esas páginas recalcará Giner el abigarramiento de concepciones contradictorias propio del eclecticismo doctrinarista, que sólo puede admitir la mínima cohesión de los planteamientos formalistas, sin trascender jamás de las cuestiones de forma.

Aun cuando no es ese su objetivo primordial, no dejará Giner de apuntar cómo el doctrinarismo permite la pervivencia de los sistemas de poder del Antiguo Régimen. Su falta de arraigo científico y popular, que obliga a «elaborar su obra en el vacío» ${ }^{19}$, con el «apoyo en las camarillas de los salones y academias», sólo engendró doctrinas jurídicas llenas de «dualidades y antítesis». Así pudo sobrevivir el «antiguo absolutismo... obligado a pactar con el liberalismo, haciéndole concesiones» ${ }^{20}$, pero, en definitiva, conservando un papel central en el concierto político. Así duró «el servilismo de la tradición y la petrificación de las costumbres» ${ }^{21}$. Así, «el doctrinarismo, con sus formas vacías y sin cuidarse para nada del fin del Estado ni de su esencial relación con la sociedad y la vida toda» ${ }^{2}$, convertía el sistema jurídico constitucional en «palenques donde los opuestos bandos se disputan ora el favor de un monarca que considera como su mejor timbre de gloria la hipócrita y perenne falsificación de un régimen incompatible con la majestad de los antiguos tronos, ora el de la opinión escéptica y egoísta de las malamente llamadas clases conservadoras...» ${ }^{23}$, el párrafo de Giner, de una rotunda y lóbrega crítica antidoctrinaria, sigue dispersándose en una cascada de entristecidas denuncias de los defectos y las sordideces que aprecia en la actitud criticada. Pero creo que con sus primeras frases, aquí reproducidas, no se podrá negar la captación por nuestro autor de que una de las consecuencias centrales del doctrinarismo fue permitir la latencia del Antiguo Régimen, al tiempo que se proclamaba su extinción.

$\mathrm{Y}$ en rigor, Giner señala como rasgos del doctrinarismo lo que hoy anunciaríamos como «consenso». Claramente escribirá que lo integran «lo mismo los hombres del altar y del trono que los que no querían trono ni altar» ${ }^{24}$. Su objetivo, que Giner estima honrado, fue «aunar la libertad con el orden, la legitimidad con la revolución, la tradición con lo por venir» ${ }^{25}$. Pero el fracaso era inevitable, pues «tan bellas palabras... no podían ser sino un lugar común en sus labios, no una realidad en sus hechos» ${ }^{20}$. Y no podía ser de otro modo por la inevitable razón de que, para lograr sus objetivos, una empresa del porte de la proclamada requiere «algo más que poner esos nombres unos al lado de otros, sin más enlace, unidad ni trascendencia». Se precisaba, y varias veces lo dirá Giner, una doctrina que se centrase sobre la idea del Estado y que «un concepto firme del Derecho diese a conocer la filiación lógica de esas ideas, su verdadero sentido y su propio lugar en el organismo político, no

19 Ibidem, pág. 77.

${ }^{20}$ Ibídem, pág. 85.

"I Ibidem, pág. 95.

22 Ibídem, pág. 113.

23 Ibidem, pág. 156.

${ }^{24}$ Ibídem, pág. 74.

${ }^{25}$ Ibídem, pág. 74.

26 Ibídem, pág. 75. 
menos que su relación esencial entre sí y con la unidad del Estado» ${ }^{27}$. En fin de cuentas, el doctrinarismo, pues, lo que no tenía era precisamente doctrina, sino sólo unos pactos que tendían a asegurar un mantenimiento de cierto statu quo, y por carecer de esa doctrina, ni podría ser un interlocutor digno con el marxismo creciente ni llegaría siquiera a poder consolidar los intereses a que se aferraba.

Es frecuente hoy por parte de los historiadores generales poner el énfasis de la explicación de las crisis decimonónicas de España, ya sea el fracaso del liberalismo, como prefiere Raymond Carr ${ }^{28}$, ya el fracaso de la revolución social española, como defiende E. J. Hobsbawm ${ }^{29}$. Pero tanto una visión como otra, que no son sino parcelas de un mismo diagnóstico, deberían ser completadas con el análisis de la terca presencia de un doctrinarismo perenne, que por sí solo explica buena parte de ambos fracasos si se presta alguna atención al testimonio de Giner, testigo especialmente cualificado para pasar a juzgar los mismos hechos que testifica.

En cierto modo resulta todavía hoy muy meditable, primero, el retorno y, luego, la conservación del doctrinarismo por el que Cánovas se decidió en la Constitución de 1876. No cabía duda de que existía ya, acabamos de verlo, un sector autorizado de opinión radicalmente contradictorio respecto de él. Las evidentes distancias ideológicas entre Giner y Cánovas no empañan el hecho que el segundo siguiese el criterio del primero, por ejemplo, respecto de cómo organizar la estructura del Senado. Así, pues, sus opiniones pesaron, pero no precisamente en un aspecto tan delicado como la condena del doctrinarismo. Por las razones que fuesen, está claro que Cánovas prefirió, o se vio obligado a preferir, una solución más de forma que de fondo.

En cualquier caso, el conservado lastre doctrinarista fue algo más que la causa formal del colapso constitucional que concluyó con la vida de la Monarquía restaurada. Puede decirse quizá que en los comienzos de la Restauración no se ofrecía otro camino que permitiese aunar voluntades en la empresa canovista. Pero el problema reside más, a mi entender, en la incesante conservación de un mecanismo constitucional doctrinario desde 1876 hasta 1931. Continuidad que resalta la carencia de la necesaria sensibilidad respecto de la exigencia de cambios durante varias generaciones de políticos. Un lento proceso de acentuación del aspecto representativo, que se desenvuelve desde la Regencia de la ejemplar reina madre doña María Cristina (1886-1902), había ido en parte actuando como mecanismo corrector de aquel doctrinarismo de los primeros momentos de 1876 , que parece puede ser calificado sin exageración como retroceso importante y equivocado, contempladas las cosas desde la perspectiva del sexenio. Pero, como ya había advertido sin éxito Giner, el doctrinarismo empujaba, por un lado, al «Estado a un grado de postración más patente que disimulado por la inestabilidad de sus instituciones» ${ }^{30} \mathrm{y}$, paralelamente, «las diversas fuerzas sociales llegaban ellas también al último límite

${ }^{27}$ Ibidem, pág. 75.

28 Raymond Carr, España 1808-1966, Barcelona, 1968.

29 E. J. Hobsbawm, Revolucionarios. Ensayos contemporáneos, Barcelona, 1978, páginas $106 \mathrm{y}$ sigs.

${ }^{30}$ Op. cit., supra, nota 8, pág. 114. 
de la opresión y de la servidumbre» ${ }^{31}$. El pacto constitucional de 1876 no dotaba al Estado de mecanismos de reacción ni frente a la «cuestión social», como entonces se dijo, ni frente a las tensiones centrífugas de tipo separatista, inútilmente intentadas remediar, por irracional reacción frente al primer republicanismo, con la reiniciación de tendencias centralizadoras. Uno de los errores de 1876 consistió en no distinguir lo profundo de lo episódico en el ensayo federalista y negar sin más el valor de la solución en sí, juzgándola en su esencia por el peso de circunstancias aleatorias, perfectamente ajenas a ella.

$\mathrm{Al}$ desatarse de nuevo la dinámica de esas contingencias en el doble frente expuesto, la convulsión social no encontró los cauces de un Estado jurídicamente trabado en sistema de poder y representación, armónicamente ordenados para ejecutar un determinado tipo de fin del Estado. Simplemente se poseía un pacto sin referenciar a tales criterios y diseñado para un objetivo histórico determinado y concreto: restaurar la Monarquía en una dinastía precisa y dentro del juego político de fuerzas existentes en 1876. Desde ahí la disociación entre los instrumentos de gobierno y las fuerzas políticas reales había ido progresivamente aumentando. Cierto que, como acaba de apuntarse, hubo tenues tendencias correctoras del punto de partida. Pero hubiese sido preciso cambiar, más que reformar, la Constitución antes de 1923, en el sentido de aproximarla a la de 1869 y en el de una más sosegada reconsideración del federalismo. Pero todos los pasos que se dieron, pasos importantes, no alcanzaron tales cotas. En 1887 se promulgó la Ley de Asociaciones, en 1888 se estableció el Jurado y en 1890 el sufragio universal. Antes y después de la Regencia también habrá rasgos, a veces acentuados, de corregir el doctrinarismo, como en la revisión de los viejos conceptos de partidos legales e ilegales. Pero lo que no admite duda es que no se dio el paso de acometer esa corrección a escala propiamente constitucional, y el sistema político de la Restauración llegó a 1923 agotadas sus posibilidades de gobierno y tuvo que romper su propia lógica interna para intentar resolver problemas de tipo cotidiano.

La crisis de 1923 ha sido expuesta por algún tratadista ilustre, como es el caso de Posada, con un cierto grado de maniqueísmo formalista ${ }^{32}$. Es evidente que el corazón del sistema previsto en la Constitución pasaba en gran medida por su artículo 32:

«Las Cortes se reúnen todos los años. Corresponde al rey convocarlas, suspender, cerrar sus sesiones y disolver simultánea o separadamente la parte electiva del Senado y el Congreso de los Diputados, con la obligación en este caso de convocar y reunir el Cuerpo o Cuerpos disueltos dentro de tres meses.»

Es cierto que, como señala Posada, el efecto del decreto que disponía cesasen en sus funciones los presidentes del Congreso y del Senado sólo podía haberse aplicado, sin romper la Constitución, durante tres meses.

Desoído el mensaje recordatorio de ese plazo que los presidentes cesados enviaron al monarca y superado el tiempo previsto en el citado artículo 32,

${ }^{31}$ Ibidem, pág. 114.

${ }^{32}$ Op. cit., supra, nota 5, págs. 302-303. 
quedaba formalmente roto el pacto constitucional establecido en el preámbulo, puesto que se suprimía por una de las instancias de poder que allí pactaban, la Corona, la presencia de la otra, es decir, las Cortes, y por un tiempo superior a los tres meses convenidos para limitar esa supresión. En un análisis solamente jurídico del problema, resulta obvio que la Monarquía había dejado de ser «constitucional» conforme al espíritu del tantas veces reproducido «Manifiesto» de Sandhurst.

Pero no es menos cierto que cabe recordar en este punto los sombríos presagios de Giner de los Ríos, que anteriormente se sintetizaron, respecto de la infecundidad del doctrinarismo para construir un Estado y para captar la presencia de nuevas fuerzas sociales, como el poderoso movimiento que daría base a la Internacional. Expresamente había señalado Giner la imposibilidad de que un planteamiento doctrinario pudiese asumir la crítica que socialismo y comunismo dirigían a su «miopía y estrechez» ${ }^{33}$. El doctrinarismo cerró puertas a necesarias integraciones de nuevas formas sociales; y prescindió hasta el gobierno de Dato de dar cauce a la revolución socialista. Y no radica tanto el fallo en los comienzos cuanto en el desarrollo de la Restauración. Recientemente se ha defendido en el montaje canovista de 1876 su establecimiento del «principio de conciliación», y eso se alega cuando desde algunos puntos de vista se han puesto de relieve con aire de crítica sus concesiones a fuetzas políticas eminentemente burguesas ${ }^{34}$. Pero repito que no es ese, a mi entender, el problema. La inicial conciliación canovista, válida, quizá inevitable en 1876, es una cosa, y otra muy diferente su estiramiento, por así decirlo, durante casi cincuenta años. Antes he hablado de su lentísimo y sobre todo desasosegado evolucionar, rehuyendo revisiones que tanto el pensamiento de Giner como la marcha de las cosas hacían urgentes. El profesor Pabón ha señalado cómo «hacia 1918, trastornada España por las repercusiones de la Gran Guerra, fue ganando el ánimo del rey la convicción pesimista de que la situación de su país difícilmente sería superada con normalidad, esto es, en el juego político establecido por la Constitución» ${ }^{35}$. Se palpaba casi la veracidad del pronóstico de Giner por quien quizá jamás había leído sus palabras al respecto pero participaba a diario en una realidad política cada vez más hundida en trampas sin salida, sumergida en lo que Carlos Seco ha llamado «la mezquina y desconcertada 'ficción' en que la estructura institucional montada por Cánovas había degenerado» ${ }^{36}$. En cualquier caso, pues, llegado 1923 , era ya muy tarde para pensar que la Constitución de 1876 podía seguir sirviendo y todo el daño consistiese, como quiere Posada, en un problema formal en torno a su artículo 32.

La Constitución de 1876 debe ser vista, en mi opinión, en predominante línea de continuidad con los esquemas aplicados en el período de «consolidación

${ }^{33}$ Op. cit., supra, nota 8, pág. 121.

${ }^{34}$ Véase el estado de las opiniones sobre el tema en Vicente Palacio Atard, La España del siglo XIX, Madrid, 1978, pág. 489. El ataque de Jordi Solé Tura y Eliseo Aja [Cons. tituciones y periodos constituyentes en España (1808-1936), Madrid, 1977] a la Constitución de 1876 es más vibrante que profundo.

${ }^{35}$ Jesús Pabón, Cambó, vol. II, parte primera, 1918-1930, Barcelona, 1969, pág. 401.

${ }^{36}$ Carlos Seco Serrano, Alfonso XIII y la crisis de la Restauración, Madrid, 1979, página 172. 
constitucional», y muy especialmente con la de 1845. Paralelamente a ese rasgo, se acentúa su discontinuidad con el espíritu del sexenio. Prácticamente insensible a cualquier sugerencia aprovechable del período republicano, la Constitución concebida para la Restauración dinástica, contrariando uno de los ejes del proyecto monárquico de Prim, sólo podía teñirse de referencias continuistas respecto de la de 1869 en algunas cuestiones muy limitadas, concretamente el asunto de las garantías personales. Así, podría establecerse que en esa materia (arts. 7, 8, 9, 14, 16 y 17 de 1876) se ha partido de la Constitución de 1845 (arts. 7, 8, 9 y 10) y se les ha dado una lectura que pasa por el espíritu de los «derechos ilegislables» que aportó la Constitución de 1869. Pero, sin negar la importancia de esa recepción, no me parece erróneo sostener que si en líneas generales el sexenio en sus ensayos monárquico y republicano planteó con hondura una reflexión sobre la idea del Estado y supone por ello una crítica de formas sociales incardinables en el Antiguo Régimen, la Constitución de 1876 encierra en su más íntima raíz significativa un intento, el más depurado si se quiere, de continuidad social del viejo sistema. No en vano la tesis de la «Constitución interna», tan rigurosamente central en el pensamiento canovista, debe mucho más de lo que habitualmente se manifiesta a Martínez Marina, pensador más retenido por el conciliarismo que influido por la Revolución ${ }^{37}$.

${ }_{37}$ José Manuel Pérez-Prendes, estudio introductorio a F. Martínez Marina, Teoría de las Cortes, Madrid, 1979. 